\title{
Melasma na gestação e suas medidas terapêuticas
}

\author{
Melasma in pregnancy and therapeutic measures \\ Melasma en el embarazo y medidas terapéuticas
}

Amanda Silva de Moraes ${ }^{1 *}$, Amanda Marques Coelho ${ }^{1}$, Daniela Flores², Giovanna Antonelli Melo Viol ${ }^{3}$, Giovanna de Carvalho Meneses Costa ${ }^{1}$, Luiza Bastos Martins ${ }^{4}$, Maria Eduarda Navarro Volpato ${ }^{5}$, Maria Júlia Ribeiro da Costa ${ }^{1}$, Vinícius Barbosa dos Santos Sales ${ }^{6}$, Carmen Déa Ribeiro de Paula7.

\begin{abstract}
RESUMO
Objetivo: Avaliar a abordagem terapêutica do melasma durante a gestação. Revisão bibliográfica: a elevação de progesterona e estrogênio durante a gravidez aumenta a expressão de enzimas melanogênicas que se associam ao surgimento do melasma. Embora o melasma não possua cura, o uso de filtros solares de amplo espectro e a não exposição excessiva ao sol são importantes medidas preventivas. Durante o prénatal, o tema costuma ser ignorado pela equipe de saúde, visto que são priorizadas outras questões da gestação, além dos altos custos dos tratamentos tópicos. Ainda assim, o melasma interfere na qualidade de vida, pois pode provocar baixa autoestima e tristeza. O tratamento desacelera a proliferação dos melanócitos e promove a degradação dos melanossomos, no entanto, tem indicação limitada na gravidez visto que essas manchas podem desaparecer até um ano após o parto. Mas, quando realizado, é prescrito o uso de medicamentos tópicos capazes de agir no clareamento da melanina, no bloqueio da função melanocítica e na eliminação dos melanócitos. Considerações finais: Visto que, as alterações hormonais, durante a gestação, estão intimamente relacionadas ao surgimento do melasma, mais estudos são necessários para garantir atenção integral à saúde da mulher nesse período.
\end{abstract}

Palavras-chave: Melanose, Gestação, Abordagem terapêutica.

\begin{abstract}
Objective: To evaluate the therapeutic approach to melasma during pregnancy. Bibliographic review: the elevation of progesterone and estrogen during pregnancy increases the expression of melanogenic enzymes, associated with the appearance of melasma. Although melasma cannot be cured, the use of broad-spectrum sunscreens and not excessive exposure to the sun are important preventive measures. During prenatal care, the theme ends up being ignored by the health team, as they focus on other issues of pregnancy, due to the high costs of topical treatments and because it is low in severity, yet melasma interferes with quality of life, as it can cause low self-esteem and sadness. Its treatment slows down the proliferation of melanocytes and promotes the degradation of melanosomes, however, it has limited indication in pregnancy since these spots can disappear up to one year after delivery. However, when performed, the use of topical medications capable of acting in the clearing of melanin, blocking melanocytic function and eliminating melanocytes is prescribed. Final considerations: Since hormonal changes during pregnancy are closely related to the onset of melasma, further studies are needed to ensure comprehensive care for women's health during this period.
\end{abstract}

Keywords: Melanosis, Pregnancy, Therapeutic approach.

${ }^{1}$ Centro Universitário do Planalto Central Apparecido dos Santos (UNICEPLAC), Brasília - DF.

*E-mail: amandaamoraeess@gmail.com

2 Faculdade de Ciências Médicas da Paraíba (FCMPB), Cabedelo - PB.

${ }^{3}$ Centro Universitário de Volta Redonda (UNIFOA), Volta Redonda - RJ.

${ }^{4}$ Universidade Federal do Sul da Bahia (UFSB), Teixeira de Freitas - BA.

${ }^{5}$ Universidade Cesumar (UniCesumar), Maringá - PR.

${ }^{6}$ Universidade Federal de Sergipe (UFS), Lagarto - SE.

7 Universidade de Brasília (UnB), Brasília - DF. 


\section{RESUMEN}

Objetivo: Evaluar el abordaje terapéutico del melasma durante el embarazo. Revisión bibliográfica: La elevación de progesterona y estrógeno durante el embarazo aumenta la expresión de enzimas melanógenas, asociadas a la aparición de melasma. Aunque el melasma no se puede curar, el uso de protectores solares de amplio espectro y no una exposición excesiva al sol son importantes medidas preventivas. Durante la atención prenatal, el tema termina siendo ignorado por el equipo de salud, ya que se enfocan en otros temas del embarazo, debido a los altos costos de los tratamientos tópicos y porque es de baja gravedad, sin embargo, el melasma interfiere con la calidad de vida, ya que puede causar baja autoestima y tristeza. Su tratamiento retarda la proliferación de melanocitos y promueve la degradación de los melanosomas, sin embargo, tiene una indicación limitada en el embarazo ya que estas manchas pueden desaparecer hasta un año después del parto. Sin embargo, cuando se realiza, se prescribe el uso de medicamentos tópicos capaces de actuar en la limpieza de la melanina, bloqueando la función melanocítica y eliminando los melanocitos. Consideraciones finales: Dado que los cambios hormonales durante el embarazo están estrechamente relacionados con la aparición del melasma, se necesitan más estudios para garantizar una atención integral de la salud de la mujer durante este período.

Palabras clave: Melanosis, Gestación, Enfoque terapéutico.

\section{INTRODUÇÃO}

A gestação é marcada por diversas mudanças imunológicas, endócrinas, metabólicas e vasculares, que exercem efeitos significativos no corpo da mulher, tornando-a mais suscetível a alterações fisiológicas ou patogênicas da pele. Na gravidez, a incidência de alterações pigmentares pode chegar a $90 \%$ e, dependendo da manifestação clínica, podem ser fisiológicas. O principal representante da hiperpigmentação localizada é o melasma, que acomete cerca de $70 \%$ das gestantes (PURIM KSM e AVELAR MFS, 2012; KRAUS AE e LEMOS F, 2019).

O melasma, também conhecido como cloasma, máscara ou pano gravídico, é uma hipermelanose cutânea crônica adquirida e caracterizada por manchas acastanhadas, irregulares e simétricas que se distribuem preferencialmente na face. É classificado conforme sua distribuição topográfica, podendo ser centrofacial ou periférico. Afeta ambos os gêneros, mas em maior proporção as mulheres na idade fértil e de pele mais pigmentada (PIRES CA e PANCOTE CG, 2017; HANDEL AC, 2013).

Dentre os fatores associados ao seu desenvolvimento, cita-se: predisposição genética, fatores hormonais (altos níveis de estrogênio, progesterona e melanocortina), gestação, uso de anticoncepcionais orais (ACO), terapia de reposição hormonal, medicamentos fotossensibilizantes, radiação ultravioleta (UV), sedentarismo, inadequação alimentar e de hidratação, fatores emocionais, cosméticos derivados do petróleo e cuidados tópicos (KRAUS AE e LEMOS F, 2019; PIRES CA e PANCOTE CG, 2017).

Em gestantes, espera-se que ocorra a resolução completa do quadro em até um ano após o nascimento da criança, porém cerca de $30 \%$ das pacientes evoluem com alguma sequela do melasma. Dessa forma, apesar de ser uma condição benigna, pode ser esteticamente significativa e comprometer o psicoemocional e social da gestante, causando um impacto negativo na qualidade de vida (PURIM KSM e AVELAR MFS, 2012; KRAUS AE e LEMOS F, 2019).

Algumas das medidas terapêuticas utilizadas durante o tratamento do melasma incluem agentes despigmentantes tópicos como a hidroquinona, o ácido azelaico, tretinoina, alfa e betahidroxiácidos, além de corticoides tópicos usados como monoterapia ou em associação. Além disso, muito frequentemente são utilizados fotoprotetores físicos, os bloqueadores solares. Esses bloqueadores não irão ser absorvidos pela pele, e vão formar um filtro que reflete e dispersa a radiação ultravioleta (UV), impedindo sua absorção. Visto que apresentam bom grau de segurança e proteção, esses fotoprotetores têm seu uso indicado às pessoas com baixo fototipo, portadores de doenças genéticas com maior risco de desenvolver cânceres de pele, residentes em áreas de maior exposição solar (como regiões intertropicais) e gestantes (PIRES CA e PANCOTE CG, 2017).

Diante do exposto, o presente estudo tem como principal objetivo avaliar as diversas alternativas de abordagem terapêutica do melasma durante o período gestacional. 


\section{REVISÃO BIBLIOGRÁFICA}

A patogênese do melasma ainda não é totalmente compreendida, mesmo com o amplo conhecimento de alguns elementos desencadeantes como a história familiar, exposição solar, gravidez, hormônios sexuais, cosméticos e medicamentos fotossensibilizantes. Sabe-se ainda que há evidente predisposição genética, visto que mais de $40 \%$ dos pacientes referem familiares acometidos pelo melasma (SHETH VM e PANDYA AG, 2011).

Durante a gravidez a produção de hormônios sexuais femininos, como progesterona e estrogênio encontra-se aumentada e esses hormônios são capazes de aumentar a expressão de algumas das enzimas melanogênicas, em especial a tirosinase e a dopacromo tautomerase (DCT). Nesse sentido, a elevação hormonal está fortemente relacionada ao surgimento da hiperpigmentação facial, principalmente durante o terceiro trimestre de gestação (MIOT LDB, et al., 2009; COSTIN GE e BIRLEA SA, 2006; PIRES CA e PANCOTE CG, 2017).

Os melanócitos são células dendríticas de origem ectodérmica responsáveis pela pigmentação da pele e, consequentemente, pela tonalidade cutânea. Essas células estão localizadas na camada basal, tendo seus dendritos estendidos ao longo de toda a extensão da epiderme atribuindo forte contato com os queratinócitos, para os quais faz a transferência da melanina. No interior desses melanócitos há os melanossomas, que são organelas elípticas altamente especializadas em sintetizar e depositar melanina. Isso ocorre porque essas organelas armazenam tirosinase, a enzima desencadeadora da produção de melanina (PAULA VG, et al., 2019).

Embora existam algumas medidas terapêuticas, o melasma não possui cura definitiva e sua recidiva é frequente. Desse modo, o objetivo principal do tratamento do melasma é promover a desaceleração da proliferação dos melanócitos e a degradação dos melanossomos (COSTIN GE e BIRLEA SA, 2006). Nessa perspectiva, a abordagem terapêutica inclui o uso de fotoproteção e despigmentantes tópicos, como o ácido azeláico e o ácido ascórbico, bem como o uso de corticoides tópicos (PIRES CA e PANCOTE CG, 2017; GAEDTKE GN, 2011).

\section{Fotoproteção}

O uso de filtros solares de amplo espectro para radiação ultravioleta $A$ e $B$ e a não exposição solar excessiva, podem prevenir o seu surgimento (PURIM KSM e AVELAR MFS, 2012; PIRES CA e PANCOTE CG, 2017; GAEDTKE GN, 2011). Nesses casos, o filtro solar deve ser aplicado meia hora antes de se expor ao sol e o reforço da proteção deve ser feito sempre que necessário. Além disso, deve-se evitar a exposição solar entre às 10 e 16 horas para que haja um menor risco de surgimento de hiperpigmentação (PURIM KSM e AVELAR MFS, 2012; GAEDTKE GN, 2011).

Os fotoprotetores químicos contêm moléculas que realizam a absorção da radiação UV e conseguem transformá-la em uma radiação de menor energia. Existem diversos grupos de filtros solares, os quais podem ser compostos de salicilatos, antranilatos, cinamatos e benzoferonas. Já os bloqueadores solares ou fotoprotetores físicos não são absorvidos pela pele e agem formando uma barreira que reflete e dispersa a radiação ultravioleta (UV), impedindo assim a sua absorção. Possuem grande espectro de proteção contra UV e podem ser compostos de dióxido de titânio e óxido de zinco (PIRES CA e PANCOTE CG, 2017; GAEDTKE GN, 2011).

Além dos protetores químicos e físicos, também existem os protetores de barreira. Roupas, boné ou chapéus, óculos de sol, guarda-sol e sombrinha ajudam a refletir os raios solares. Salienta-se que é preferível que estes objetos sejam feitos com materiais sintéticos, já que tecidos naturais não são tão eficientes na proteção contra os raios UV (PURIM KSM e AVELAR MFS, 2012; PIRES CA e PANCOTE CG, 2017; GAEDTKE GN, 2011; SEHGAL VN, et al., 2011).

\section{Prevalência}

Um estudo realizado com 224 gestantes em Porto Alegre observou uma prevalência de 10\% de melasma entre as 224 gestantes avaliadas. Os autores relacionaram a ocorrência a algumas variáveis, identificando a existência de forte associação apenas com a idade, provavelmente por maior tempo de exposição aos fatores 
de risco e alterações hormonais. Nenhuma relação significativa foi observada entre o melasma e a etnia, idade gestacional e fototipos de pele de acordo com a classificação de Fitzpatrick. Também não foi evidenciada relação com pacientes que faziam uso anterior de anticoncepcionais. Os pesquisadores concluíram, ainda, que as participantes não sabiam como prevenir o aparecimento e a evolução do melasma, ou seja, relatando pouco conhecimento sobre a doença (HEXSEL D, et al., 2009).

Um outro estudo, realizado no Irã, encontrou uma prevalência de 15,8\% entre as gestantes avaliadas. Neste trabalho evidenciou história familiar em $54 \%$ dos casos, além da associação do melasma com etnia, fototipo e número de paridade (MOIN A, et al., 2006). Já em um estudo realizado em unidades básicas de saúde na cidade de São Paulo revelou o quanto o tema é ignorado pelas equipes de saúde. Os profissionais relataram abordar poucas vezes ou nunca o problema durante as consultas, visto que, durante o decorrer do pré-natal, valorizam outras questões da gestação, do puerpério e também dos recém-nascidos. Algumas das dificuldades foram justificadas pelos altos custos dos tratamentos tópicos, a não percepção da necessidade da paciente, o pouco conhecimento do profissional e o tempo limitado para cada atendimento (URASAKI MBM, 2018).

Acredita-se que a pouca atenção dada ao melasma ocorre também devido a sua pouca gravidade e baixa morbidade, apesar de sua alta incidência. Somado a isso, há desconhecimento pelos profissionais de saúde sobre a doença em si e as suas consequências psicossociais em relação à paciente acometida. Em contrapartida, quando as pacientes são avaliadas, compreende-se o quanto a patologia tem significado impactante em suas vidas, provocando baixa autoestima, vergonha, raiva e tristeza. Algumas relatam dificuldades financeiras para o tratamento e, por isso, camuflam as manchas com o uso de maquiagem. Enfim, as máculas implicam em queda na qualidade de vida, uma vez que acabam interferindo nas esferas sociais, na autoimagem e causando sofrimento psíquico (URASAKI MBM, et al., 2013).

\section{Fatores de risco}

A exposição solar, mas não a queimadura, é o fator desencadeante mais importante. A radiação ultravioleta aumenta diretamente a atividade melanogênica, provocando a pigmentação epidérmica mais intensamente nas regiões já acometidas do que na pele adjacente (HANDEL AC, 2013). Além disso, foi demonstrado que os comprimentos de onda mais curtos da luz visível (luz azul-violeta) induzem uma hiperpigmentação através de um sensor específico presente nos melanócitos chamado opsina 3 (PASSERON T e PICARDO M, 2018).

Um trabalho realizado nos EUA em 1967, com 61 mulheres que desenvolveram melasma decorrente do uso de anticoncepcionais orais, verificou que 52 (87\%) também o referiram na gestação. Este dado indica que um evento pigmentar induzido por hormônio sexual pode ser fator de risco para outro evento subsequente, em indivíduos com certa predisposição (HANDEL AC, 2013). Um estudo indiano que comparou FSH, LH, prolactina, estrogênio e progesterona entre 36 mulheres com melasma e controles da mesma idade observou que houve diferença nos níveis de 17-ü-estradiol no início do ciclo menstrual entre os grupos, sugerindo que estrogênios circulantes possam ser fatores de risco e mantenedores da doença (HASSAN I, et al., 1998).

$\mathrm{Na}$ gravidez, especialmente no terceiro trimestre, há estímulo para a melanogênese e o aumento dos hormônios placentários, ovarianos e pituitários podem justificar a ocorrência do melasma gravídico. A elevação do hormônio estimulante de melanócitos (MSH), estrogênio e progesterona também levam ao aumento da transcrição de tirosinase e dopacromo tautomerase, o que pode estar diretamente envolvido no desenvolvimento da pigmentação nessa fase (HANDEL AC, 2013). Como os melanócitos contêm ambos os receptores para estrogênio: citosólico e nuclear, é possível que tais células, nas pacientes que desenvolveram melasma, sejam inerentemente mais sensíveis aos efeitos estimulantes dos estrogênios e outros hormônios esteróides sexuais (GOGLIA L, et al., 2014).

O uso de cosméticos e a ingestão de certos medicamentos, como anticonvulsivantes e outras substâncias fotossensibilizantes, também foram apontados como fatores de risco para melasma. Da mesma forma, uma ampla variedade de produtos químicos como arsênio, ferro, cobre, bismuto, prata, ouro; e drogas como antimaláricos, tetraciclinas, anticonvulsivantes, amiodarona, sulfonilureias, entre outros, podem causar hiperpigmentação da pele, por serem capazes de realizar deposição nas camadas superficiais ou por estímulo 
à melanogênese (HANDEL AC, 2013). Já a pesquisa realizada por Sanchez com 76 pacientes porto riquenhas, não encontrou associação entre a doença e o uso de qualquer produto químico, sugerindo que, embora possível, não sejam as exposições químicas exógenas o principal agente etiológico da doença (ELLING SV e POWELL FC, 1997; SANCHEZ NP, et al., 1981).

\section{Tratamento}

Na prática, o tratamento durante a gravidez não é frequentemente indicado e isso se deve ao fato de o melasma desaparecer até um ano após o parto na maioria dos casos. Evidencia-se ainda o cuidado da maioria dos profissionais em não oferecer medicamentos que podem trazer riscos à gestante, visto que estes podem não ser totalmente seguros, como demonstrado em alguns estudos (GAEDTKE GN, 2011). Nesse sentido, os médicos assumem uma abordagem cautelosa quanto ao tratamento no período gravídico, adiando a terapia até o período pós-parto, tendo em vista que as próprias gestantes estão de acordo com tal conduta (NUSSBAUM R e BENEDETTO AV, 2006).

Os medicamentos tópicos podem agir no clareamento da melanina, no bloqueio da função melanocítica e na eliminação dos melanócitos. As drogas que são consideradas eficazes e seguras para o melasma na gestante são o ácido azeláico e o aloe vera encapsulado em lipossomas. Já os que não podem ser utilizados são a hidroquinona e os retinóides. Independente do tratamento escolhido, é essencial evitar a exposição às radiações solares para que o mesmo seja eficaz (KRAUS AE e LEMOS F, 2019; GAEDTKE GN, 2011).

O ácido azelaico, considerado categoria B pela Food and Drug Administration (FDA), demonstra relativa segurança materno-fetal quando aplicado topicamente, por reduzir secreção de metaloproteinases e de fatores de crescimento como HGF e SCF, os quais são promelanogênicos. Dessa forma, tal fármaco representa uma boa estratégia terapêutica devido à possibilidade de atuar no mecanismo de ação de receptores ativados por proliferadores de peroxissoma tipo gama (PPARY), que estão envolvidos na modulação da resposta melanocítica na regulação da pigmentação (COUTINHO GSL, et al., 2012; PASSERON T e PICARDO M, 2018; MARESCA V, et al., 2015).

No que se diz respeito à hidroquinona, observa-se que seu mecanismo de ação consiste em diminuir a produção de melanina por meio da inibição da tirosinase, além de atuar na morfologia dos melanócitos, induzindo, portanto, a degradação dos melanossomas. No entanto, independente da concentração administrada, irritações cutâneas associadas à hiperpigmentação pós-inflamatória secundária e ocronose exógena irreversível podem surgir após o uso (BOLANČA I, et al., 2008; NASCIMENTO DB, et al., 2017).

O extrato da uva-ursina pode ser utilizado como componente do Skin Whitening Complex (SWC), em conjunto com Aspergillus biofermentado, extrato de grapefuit e o extrato de arroz. Tal composto, administrado em concentrações de 2 a 5\% revela semelhante significância terapêutica quando comparado com a hidroquinona, podendo ser realizada tal terapia em casos de contraindicação de outros tópicos despigmentantes, visto que não foram relatados efeitos colaterais nem mesmo para pacientes gestantes. (MOREIRA AM, et al., 2010). Também não foram observados efeitos irritantes (NASCIMENTO DB, et al., 2017).

Ainda que os agentes clareadores tópicos sejam considerados padrão-ouro no tratamento do melasma, suas limitações abrangem questões que dependem de como a fisiopatologia dessa dermatose é discutida. Nesse sentido, é essencial adotar uma abordagem holística e reconhecer que se trata também de distúrbio de fotoenvelhecimento, já que os tratamentos associados à fotoproteção com alto índice de fator de proteção solar não são totalmente capazes de eliminar as lesões e evitar o reaparecimento das manchas (PASSERON T e PICARDO M, 2018).

Os resultados apresentados após o uso de extrato em gel de aloe vera encapsulado em lipossomas foi superior ao do uso de ácidos graxos essenciais na diminuição da gravidade do melasma durante a gravidez devido à sua facilidade na percolação. $O$ extrato é capaz de aliviar a mancha com efeitos colaterais leves e isso se deve ao fato de a babosa ser rica em minerais, vitaminas e aminoácidos. Sendo assim, o aloe vera pode ser considerado uma opção terapêutica para o melasma durante a gravidez (GHAFARZADEH M e EATEMADI A, 2017). 
O ácido ascórbico é outra opção de agente despigmentante para a realização do tratamento. Este age impedindo a melanogênese através da inibição da tirosinase, além de ter efeito anti-radical livre. Todavia, o seu efeito é transitório e limitado em fase aquosa, já que o mesmo sofre oxidação rapidamente e tem dificuldade para penetrar na pele. Por conta disso, foi desenvolvido o fosfato de ascorbil magnésio (VC-PMG), com grande estabilidade em solução aquosa e boa penetração cutânea (GAEDTKE GN, 2011; PIRES CA e PANCOTE CG, 2017; NASCIMENTO DB, et al., 2019).

O uso do VC-PMG por 2 a 3 meses nas concentrações de 1 a $3 \%$ demonstrou resultados bastante satisfatórios e também funcionou bem como um agente anti-envelhecimento. No entanto, sua capacidade de clareamento cutâneo é leve e, por isso, é frequentemente utilizado na fase de manutenção ou combinado a outros despigmentantes no tratamento do melasma. O uso do ácido ascórbico e seus derivados durante a gestação permanece controverso, visto que, atualmente, estes ainda não foram classificados pela categorização do FDA (GAEDTKE GN, 2011; PIRES CA e PANCOTE CG, 2017).

O Ácido Kójico também tem sido utilizado como uma opção de tratamento tópico por agir de maneira segura e não ser citotóxico, não provocando irritações. Sua ação clareadora consiste em inibir a formação da melanina, principalmente, através da inibição da enzima tirosinase por meio da quelação do íon cobre, o que acaba impedindo a oxidação necessária para produção da melanina (PAULA VG, et al., 2019).

Além da importância do protetor solar como forma de prevenção ao surgimento do melasma, durante o tratamento com o Ácido Kójico, pode haver esfoliação da pele e, devido a isso, o uso do protetor solar tornase ainda mais indispensável durante todo o tratamento. No entanto, esse ácido é um despigmentante com ação mais suave que os demais geralmente prescritos, além de não causar fotossensibilização na paciente permitindo com que ela o utilize durante o dia (PAULA VG, et al., 2019).

A tretinoína é um derivado da vitamina $A$ que é frequentemente utilizado no tratamento, porém se torna mais eficaz quando combinado com hidroquinona e corticosteróides. A concentração utilizada é de 0,05 a 1\%. Porém, é classificada pela Food and Drug Administration como risco $\mathrm{C}$ em relação à gestação, podendo causar irritação da pele, fotossensibilidade e hiperpigmentação paradoxal. No entanto, o uso durante a amamentação pode ser considerado seguro (GAEDTKE GN, 2011; KRAUS AE e LEMOS F, 2009).

Os alfa-hidroxiácidos (AHA), componentes de alguns cosméticos e de produtos naturais, são classificados pela FDA como risco $B$, sendo considerado seguro de acordo com a toxicidade para o lactente. Tais agentes com pH menor ou igual a 3,5 e concentração menor ou igual a 10\% revelam-se como outra medida terapêutica para as gestantes, visto que podem estimular a proliferação celular da epiderme e reduzir a espessura da pele, tornando-a mais flexível, por meio da redução da adesão dos corneócitos no estrato córneo, em conjunto com a síntese de colágeno, estimulada pelos glicosaminoglicanos. Este mecanismo contribui para diminuição de rugas e homogeneidade da pigmentação, sendo relatado benefícios na textura da pele após o uso (FIGUEIRÓ TLM, et al., 2008).

Dentro dos despigmentantes, o cosmocaíne, obtido pelo gérmen do trigo, apresenta-se relevante no tratamento do melasma gravídico uma vez que regula a melanogênese de uma forma não agressiva, induzindo à estimulação para formação de pigmentos claros. Além disso, não se configura como citotóxico nem hipopigmentante, o que oferece segurança no uso para gestantes (FIGUEIRÓ TLM, et al., 2008).

Corticóides tópicos inibem a síntese de melanina por meio da supressão da atividade dos melanócitos, mas sem causar a sua destruição. No entanto, como têm efeito despigmentante leve, geralmente são utilizados em combinação com tretinoína e hidroquinona. Essa associação diminui o efeito irritativo provocado pelos compostos ácidos, além de diminuir a melanogênese em 8 semanas. Ademais, corticóides de alta potência, como o propionato de clobetasol, podem ser utilizados isoladamente, mas o efeito clareador não se mantém após a sua suspensão (SEHGAL VN, et al., 2011; PIRES CA e PANCOTE CG, 2017; BOLANČA I, et al., 2008; GAEDTKE GN, 2011; PASSERON T e PICARDO M, 2018).

Devido à dificuldade nos processos terapêuticos, o que costuma ser mais eficiente são as terapias combinadas, de forma que cada produto utilizado aja de uma maneira específica em cada etapa da melanogênese, resultando em resultados mais expressivos (PAULA VG, et al., 2019). 


\section{CONSIDERAÇÕES FINAIS}

Apesar do melasma ser uma condição de pouca gravidade e baixa morbidade, apresenta alta incidência e pouco conhecimento pelos profissionais de saúde. Sabe-se da associação com a elevação hormonal, principalmente ao final da gestação, e embora existam várias opções de tratamento, não possui cura definitiva. A prevenção deve ser indicada com uso de filtros solares de amplo espectro e com a não exposição solar excessiva. Apesar disso, o tratamento durante a gravidez é limitado, e, quando realizado, os médicos indicam uma abordagem cautelosa. Assim, conclui-se que, como a elevação hormonal que ocorre durante a gravidez está relacionada ao surgimento da hiperpigmentação facial, mais estudos a respeito do tema precisam ser realizados, para que as pacientes tenham uma atenção integral à saúde durante esse período.

\section{REFERÊNCIAS}

1. BOLANČA I, et al. Chloasma - The Mask of Pregnancy. Collegium Antropologicum, 2008; 32(2):139-141.

2. CARIO M. How hormones may modulate human skin pigmentation in melasma: an in vitro perspective. Experimental dermatology, 2019; 28(6):709-718

3. COSTIN GE, BIRLEA SA. What is the mechanism for melasma that so commonly accompanies human pregnancy?. International Union of Biochemistry and Molecular Biology Life, 2006; 58(2): 55-57.

4. COUTINHO GSL, et al. Prescrição de produtos dermocosméticos durante a gravidez. Ciência \& Saúde, 2012; 5(1):1625.

5. ELLING SV, POWELL FC. Physiological changes in the skin during pregnancy. Clinics in Dermatology, 1997. Volume 15, Issue 1: 35-43.

6. FIGUEIRÓ TLM, et al. Pele e gestação: aspectos atuais dos tratamentos e drogas comumente utilizados - Parte I. Femina, 2008; 36(8):511-521.

7. GAEDTKE GN. Abordagem Terapêutica do Melasma na Gestação - Revisão Bibliográfica. Trabalho de Conclusão de Curso (Pós-Graduação em Medicina Estética) - Instituto Paulista de Ensino em Medicina e Cirurgia Estética. Universidade Tuiuti Do Paraná, Curitiba, 2011;22 p.

8. GHAFARZADEH M, EATEMADLI A. Clinical efficacy of liposome-encapsulated Aloe vera on melasma treatment during pregnancy. Journal of Cosmetic Laser Therapy. 2017;19(3):181-187.

9. GOGLIA L, et al. Melasma: A Cosmetic Stigma During Pregnancy. Journal of Pigmentary Disorders, $2014 ; 1-4$.

10. HANDEL AC. Fatores de Risco para Melasma Facial em Mulheres: um Estudo Caso-Controle. Dissertação (Mestrado em Patologia) - Faculdade de Medicina. Universidade Estadual Paulista, Botucatu, 2013; 100 p.

11. HASSAN I, et al. Hormonal milieu in the maintenance of melasma in fertile women. The Journal of dermatology, 1998; 25(8):510-512.

12. HEXSEL D, et al. Melasma and pregnancy in southern Brazil. Journal of the European Academy of Dermatology and Venereology, 2009; 23(3):367-368.

13. KRAUS AE, LEMOS F. Abordagem Terapêutica do Melasma no Período Gestacional: Revisão de Literatura. Trabalho de Conclusão de Curso (Graduação em Cosmetologia e Estética). Universidade do Sul de Santa Catarina, Palhoça, 2019; $21 \mathrm{p}$.

14. MARESCA V, et al. Skin phototype: a new perspective. Pigment cell \& melanoma research, 2015; 28(4):378-389.

15. MIOT LDB, et al. Fisiopatologia do melasma. Anais Brasileiros de Dermatologia, 2009; 84(6):623-635.

16. MOIN A, et al. Prevalence and awareness of melasma during pregnancy. International Journal of Dermatology, 2006; 45(3):285-288.

17. MOREIRA AM, et al. Estudo duplo cego comparativo entre hidroquinona e extrato de uva-ursina no tratamento do melasma. Surgical \& Cosmetic Dermatology, 2010; 2(2):99-104.

18. NASCIMENTO DB, et al. Etiologia e tratamento medicamentoso de melasmas durante a gestação. Revista de Iniciação Científica e Revisão, 2019; 2(3):176-180.

19. NUSSBAUM R, BENEDETTO AV. Cosmetic aspects of pregnancy. Clinics in Dermatology, 2006; 24(2):133-141.

20. PASSERON T, PICARDO M. Melasma, a photoaging disorder. Pigment cell \& melanoma research, 2018; 31(4):461465.

21. PAULA VG, et al. Contribuições do farmacêutico defronte a atenuação do melasma durante e depois da gestação. Revista JRG de Estudos Acadêmicos, 2019; 2(4):220-235.

22. PIRES CA, PANCOTE CG. Prevenção e Tratamento do Melasma na Gestação. Revista Corpus Hippocraticum, 2017; $1: 1-11$.

23. PURIM KSM, AVELAR MFS. Fotoproteção, melasma e qualidade de vida em gestantes. Revista Brasileira de Ginecologia e Obstetrícia, 2012; 34(5):228-234.

24. SANCHEZ NP, et al. Melasma: a clinical, light microscopic, ultrastructural, and immunofluorescence study. Journal of the American Academy of Dermatology, 1981; 4(6):698-710.

25. SEHGAL VN, et al. Melasma: Treatment strategy. Journal of Cosmetic and Laser Therapy, 2011; 13(6):265-279.

26. SHETH VM, PANDYA AG. Melasma: a comprehensive update: part I. Journal of the American Academy of Dermatology, 2011; 65(4):689-697.

27. URASAKI MBM, et al. Impactos psicossociais associados às manchas gravídicas. Cogitare Enfermagem, 2013; 18(4):655-662.

28. URASAKI MBM. Conhecimento, atitude e prática da equipe de saúde sobre melasma na gravidez. Avances en Enfermería, 2018; 36(1):40-49. 